\title{
Tensor-Based Angle and Range Estimation Method in Monostatic FDA-MIMO Radar
}

\author{
Tengxian Xu, ${ }^{1}$ Yongqin Yang $\mathbb{D},{ }^{1}$ Mengxing Huang $\left(\mathbb{D},{ }^{1}\right.$ Han Wang, ${ }^{2}$ Di Wu, ${ }^{1}$ and Qu Yi ${ }^{3}$ \\ ${ }^{1}$ State Key Laboratory of Marine Resource Utilization in South China Sea, \\ School of Information and Communication Engineering, Haikou 570228, China \\ ${ }^{2}$ College of Physical Science and Technology, Yichun University, Yichun 336000, China \\ ${ }^{3}$ College of Physics and Electronic Engineering, Hainan Normal University, Haikou 571158, China
}

Correspondence should be addressed to Yongqin Yang; yangyq@hainanu.edu.cn and Mengxing Huang; huangmx09@163.com

Received 3 July 2020; Accepted 15 July 2020; Published 12 August 2020

Guest Editor: Wang Zheng

Copyright (c) 2020 Tengxian Xu et al. This is an open access article distributed under the Creative Commons Attribution License, which permits unrestricted use, distribution, and reproduction in any medium, provided the original work is properly cited.

\begin{abstract}
In the paper, joint angle and range estimation issue for monostatic frequency diverse array multiple-input multiple-output (FDAMIMO) is proposed, and a tensor-based framework is addressed to solve it. The proposed method exploits the multidimensional structure of matched filters in FDA-MIMO radar. Firstly, stack the received data to form a third-order tensor so that the multidimensional structure information of the received data can be acquired. Then, the steering matrices contain the angle and rang information are estimated by using the parallel factor (PARAFAC) decomposition. Finally, the angle and range are achieved by utilizing the phase characteristic of the steering matrices. Due to exploiting the multidimensional structure of the received data to further suppress the effect of noise, the proposed method performs better in angle and range estimation than the existing algorithms based on ESPRIT, simulation results can prove the proposed method's effectiveness.
\end{abstract}

\section{Introduction}

Multiple-input multiple-output (MIMO) radar was first proposed in [1-3], which is a key research point in today's radar field. In MIMO radar, all the antennas are omnidirectional and the transmitted signals are orthogonal to each other, which can achieve the waveform diversity gain in the receiver side. Currently, there are two main types of MIMO radars, namely, collocated MIMO radar [4-6] and statistical MIMO radar [7, 8]. The statistical MIMO radar is composed with separated transmit and receive antennas for obtaining both the waveform and spatial diversity gain. In contrast, in order to improve the estimation performance, the collocated MIMO radar closely places the transmitting and receiving arrays to form a virtual array that has large aperture. In the past few years, parameter estimation, including direction of arrival (DOA), direction of departure (DOD), and Doppler frequency, became a hot topic and investigated by a lot of researchers. In the common subspace-based methods, such as multiple signal classification algorithm (MUSIC) and estimation of signal parameters via rotational invariance techniques (ESPRIT), MIMO radar has used them to achieve DOD and DOA estimation [9-14], which can obtain the desired performance with reasonable SNR and snapshots. In addition, a joint angle and Doppler frequency estimation method is investigated, which can be used to track the targets. However, it is noted that these methods for MIMO radar with narrow-band signals cannot achieve the rang information which is very important for target localization in practice.

FDA radar can get the distance information of the target [15]. There is one difference between the FDA radar and the traditional phased array radar, and for the FDA radar, the transmitting frequency of each transmitting antenna has a frequency increment so that it can draw the relevant beam pattern, improve the degree of freedom in space, and then achieve the joint angle and distance estimation about target in the FDA radar. However, the angle and range of the target in the beam domain of the FDA radar are mixed, so it cannot directly obtain the angle and distance information of the 
target through spectral peak search. At present, there are some solutions to this problem. In [16], a nonlinear FDA radar is proposed, but this method needs to change the array element position in actual operation, which is difficult to achieve. In $[17,18]$, a method based on subarrays to achieve angle and distance decoupling is proposed, but this method has the problem of distance ambiguity. In addition, in [19], the target positioning method of the coprime array FDA radar is studied, and in $[20,21]$, the nested FDA parameter estimation method is studied.

In [18], FDA-MIMO radar with both frequency and waveform diversity is proposed for the first time, which makes use of the unique advantages of FDA radar and MIMO radar, which not only has excellent target detection performance in target detection but also has a high freedom of the spatial degree; it can realize the joint estimation of the target angle and range [17, 22-24]. At present, there are many kinds of FDA-MIMO radar target positioning algorithms, such as MUSIC methods and ESPRIT methods, but the MUSIC method involves a spectral peak search and results in a high algorithm complexity. Although the ESPRIT method can be applied to reduce the computational complexity and obtain the angle and range information of the target through rotation invariance, this method is not suitable for the phase ambiguity situation.

The advantages of FDA are developed based on the characteristics of the multidimensional structure of the signal. The abovementioned traditional matrix processing method cannot make good use of the multidimensional characteristics, which limits their performance [25]. To take advantage of the multidimensional structural characteristics of the signal, the PARAFAC decomposition is used for angle estimation in unknown target localization by modelling tensor signals [26-28]. At present, there are few studies on tensor-based FDA MIMO target estimation methods. The paper proposes the algorithm based on parallel factorization in monostatic FDA-MIMO radar. This method first establishes a third-order tensor signal model and uses parallel factorization to obtain the transmission direction matrix and the reception direction matrix $[29,30]$. Then, obtain the angle and distance information of the target by taking the phase of the direction matrix and finally realize the angle and distance estimation.

The proposed method compares the achievement with traditional estimation of signal parameters via rotational invariance techniques (ESPRIT) method [31], Unitary ESPRIT method [32], and Cramér-Rao Bound (CRB). The paper structure is divided into multiple parts. Section 2 introduces the tensor-based data model. Section 3 deduces the method of angle estimation and distance estimation. The $\mathrm{CRB}$ of the target estimation method in FDA-MIMO radar is shown in Section 4. The Section 5 shows the simulation results.

Notation: $\otimes, \odot$, and ${ }^{\circ}$ represent the Kronecker product, Khatri-Rao product, and Hadamard product, respectively. $(\cdot)^{T},(\cdot)^{H},(\cdot)^{-1},(\cdot)^{*}$, and $(\cdot)^{+}$stand for transpose, conjugate-transpose, inverse, conjugate, and pseudoinverse, respectively. $\mathbf{D}_{n}(\mathbf{A})$ represents a diagonal matrix, which composed by the $n$th row of A. $\|\cdot\|_{F}$ represents the Frobenius norm. $\mathbf{I}_{K}$ indicates a $K \times K$ identity matrix. angle $(\cdot)$ and $\min (\cdot)$ represent the phase angle of each element and the minimum element in the array. $\operatorname{Re}(\cdot)$ denotes the real part operator. $\mathbf{x}_{m, n, l}$ is denoted as a third-order tensor.

\section{Tensor-Based Data Model of Monostatic FDA- MIMO Radar}

The paper is based on a narrowband monostatic FDAMIMO radar. It is composed by $M$-element transmitting antennas and $N$-element receiving antennas. Figure 1 shows the structure of monostatic FDA-MIMO radar. The transmitting array and receiving array are placed together so the direction of arrival (DOA) is the same as the direction of departure (DOD). In general, the transmitting array and receiving array are uniform linear arrays (ULA) and are separated by half a wavelength. In the paper, there are $K$ farfield targets by default which are independent of each other. Based on the first element of the transmitting antenna, the frequency of the $m$ th element transmitting antenna is derived as

$$
f_{m}=f_{0}+(m-1) \Delta f
$$

where the reference frequency is denoted by $f_{0}$ and $\Delta f$ denotes frequency increase of the transmitting antenna. Moreover, $\Delta f \ll f_{0}$.

After the matched filter, the receiving data can be rearranged as

$$
\begin{aligned}
\mathbf{x}(t)= & {\left[\mathbf{a}_{t}\left(\theta_{1}, r_{1}\right) \otimes \mathbf{a}_{r}\left(\theta_{1}\right), \mathbf{a}_{t}\left(\theta_{2}, r_{2}\right) \otimes \mathbf{a}_{r}\left(\theta_{2}\right), \ldots, \mathbf{a}_{t}\left(\theta_{k}, r_{k}\right)\right.} \\
& \left.\otimes \mathbf{a}_{r}\left(\theta_{k}\right)\right] \mathbf{s}(t)+\mathbf{n}(t),
\end{aligned}
$$

where $\mathbf{n}(t)$ denotes an $M N \times 1$ noise vector that is the additional white Gaussian noise vector, $\quad \mathbf{a}_{t}\left(\theta_{k}, r_{k}\right)=\left[1, e^{\left(j 2 \pi\left(d / \lambda_{0}\right) \sin \left(\theta_{k}\right)-j 2 \pi(2 \Delta f / c) r_{k}\right)}, \ldots\right.$, $\left.e^{\left(j 2 \pi\left(d / \lambda_{0}\right)(M-1) \sin \left(\theta_{k}\right)-j 2 \pi(2 \Delta f / c)(M-1) r_{k}\right)}\right]^{T}$ and $\mathbf{a}_{r}\left(\theta_{k}\right)=[1$, $\left.e^{\left(j 2 \pi f_{0} d \sin \left(\theta_{k} / c\right)\right)}, \ldots, e^{\left(j 2 \pi f_{0}(N-1) d \sin \left(\theta_{k} / c\right)\right)}\right]^{T}$ stand for the transmit steering vector and the receive steering vector, respectively, and $\mathbf{X}=[x(1), x(2), \ldots, x(L)]$ stands for the received data of $L$ samples, which can be expressed as

$$
\mathbf{X}=\left[\begin{array}{c}
\mathbf{X}_{1} \\
\mathbf{X}_{2} \\
\vdots \\
\mathbf{X}_{N}
\end{array}\right]=\left[\mathbf{A}_{T} \odot \mathbf{A}_{R}\right] \mathbf{B}^{T}=\left[\begin{array}{c}
\mathbf{A}_{R} \mathbf{D}_{1}\left(\mathbf{A}_{T}\right) \\
\mathbf{A}_{R} \mathbf{D}_{2}\left(\mathbf{A}_{T}\right) \\
\vdots \\
\mathbf{A}_{R} \mathbf{D}_{N}\left(\mathbf{A}_{T}\right)
\end{array}\right] \mathbf{B}^{T}
$$

where $\mathbf{B}=[\mathbf{b}(1), \mathbf{b}(2), \ldots, \mathbf{b}(L)]^{T} \in \mathbb{C}^{L \times K}$, the transmitting direction matrix is defined as $\mathbf{A}_{T}=\left[\mathbf{a}_{t}\left(\theta_{1}, r_{1}\right)\right.$, $\left.\mathbf{a}_{t}\left(\theta_{2}, r_{2}\right), \ldots, \mathbf{a}_{t}\left(\theta_{k}, r_{k}\right)\right] \in \mathbb{C}^{M \times K}$, and the receiving direction matrix is defined as $\mathbf{A}_{R}=\left[\mathbf{a}_{r}\left(\theta_{1}\right), \mathbf{a}_{r}\left(\theta_{2}\right), \ldots\right.$, $\left.\mathbf{a}_{r}\left(\theta_{k}\right)\right] \in \mathbb{C}^{N \times K} . \mathbf{A}_{T} \odot \mathbf{A}_{R}$ stands for Khatri-Rao product. So, $\mathbf{X}_{n}$ is expressed as

$$
\mathbf{X}_{n}=\mathbf{A}_{R} \mathbf{D}_{n}\left(\mathbf{A}_{T}\right) \mathbf{B}^{T}, \quad n=1,2, \ldots, N .
$$

Under the interference of noise, the signal model is updated as 


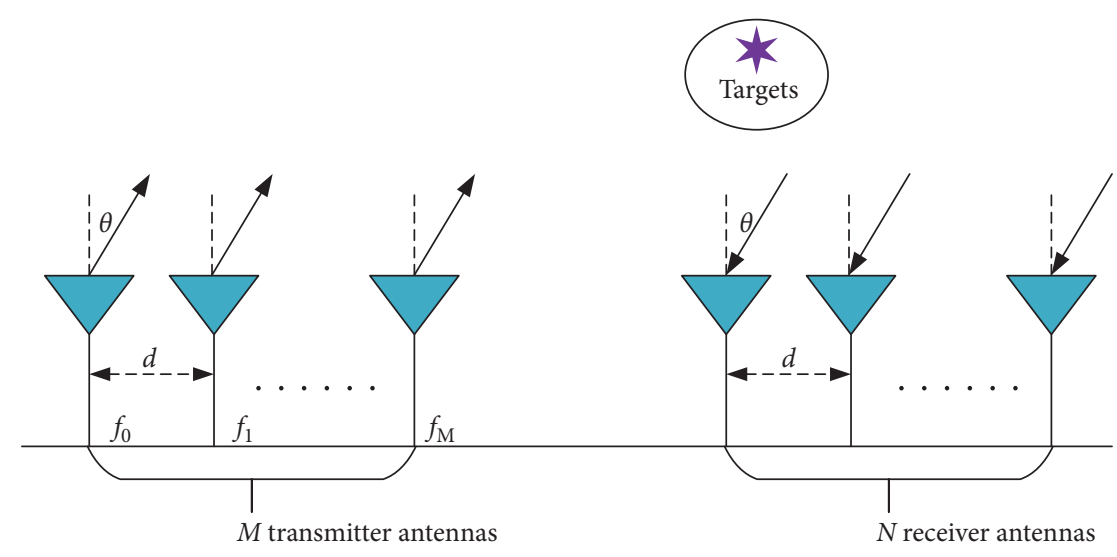

FIgURe 1: Monostatic FDA-MIMO radar.

$$
\widetilde{X}_{n}=\mathbf{A}_{R} \mathbf{D}_{n}\left(\mathbf{A}_{T}\right) \mathbf{B}^{T}+\mathbf{V}_{n}, \quad n=1,2, \ldots, N
$$

$$
\mathbf{x}_{m, n, l}=\sum_{k=1}^{K} \mathbf{A}_{R}(n, k) \mathbf{A}_{T}(m, k) \mathbf{B}(l, k)
$$

$m=1, \ldots, M, n=1, \ldots, N, l=1, \ldots, L$

where $\mathbf{V}_{n}$ stands for the received noise of the $n$th slice. By the characteristics of the tensor model, equation (4) is expanded by where $\mathbf{A}_{T}(m, k)$ is defined as the $(m, k)$ element of the matrix $\mathbf{A}_{T}$ and $\mathbf{A}_{R}(n, k)$ is defined as the $(n, k)$ element of the matrix $\mathbf{A}_{R}$. Another term for the PARAFAC decomposition of a third-order tensor is trilinear decomposition. Figure 2 shows the specific decomposition process.

Under the definition of PARAFAC decomposition, equation (6) can be regarded as a trilinear model with symmetrical properties. So, we can get $\mathbf{Y}_{m}=$ $\mathbf{B D}_{m}\left(\mathbf{A}_{R}\right) \mathbf{A}_{T}^{T}, m=1,2, \ldots, M \quad$ and $\quad \mathbf{Z}_{l}=\mathbf{A}_{T} \mathbf{D}_{l}(\mathbf{B}) \mathbf{A}_{R}^{T}$, $l=1,2, \ldots, L$. By the symmetry of the trilinear decomposition, the matrices $\mathbf{Y}$ and $\mathbf{Z}$ are constructed as

$$
\begin{aligned}
& \mathbf{Y}=\left[\begin{array}{c}
\mathbf{Y}_{1} \\
\mathbf{Y}_{2} \\
\vdots \\
\mathbf{Y}_{M}
\end{array}\right]=\left[\mathbf{A}_{R} \odot \mathbf{B}\right] \mathbf{A}_{T}^{T}, \\
& \mathbf{Z}=\left[\begin{array}{c}
\mathbf{Z}_{1} \\
\mathbf{Z}_{2} \\
\vdots \\
\mathbf{Z}_{L}
\end{array}\right]=\left[\mathbf{B} \odot \mathbf{A}_{T}\right] \mathbf{A}_{R}^{T} .
\end{aligned}
$$

We apply the tensor signal model and parallel factor decomposition to the monostatic FDA-MIMO radar and derive the PARAFAC-based angle and range estimation method. In the following section, we will derive the method in more detail.

\section{The Proposed Method}

3.1. Parallel Factor Decomposition. In this section, according to the trilinear alternating least square (TALS) method, we can estimate the transmitting direction matrix and the receiving direction matrix. The TALS method can be explored for data analysis in trilinear models.

According to [33], the estimation of the direction matrices can be obtained by the parallel factor decomposition. The least squares (LS) update of equation (3) is written by

$$
\min _{\mathbf{A}_{T}, \mathbf{A}_{R}, \mathbf{B}}\left\|\tilde{X}-\left[\mathbf{A}_{T} \odot \mathbf{A}_{R}\right] \mathbf{B}^{T}\right\|_{F}
$$

where the noise signal is denoted by $\tilde{X}$. The LS update of matrix $\mathbf{B}$ is written by

$$
\widehat{B}^{T}=\left[\widehat{A}_{T} \odot \widehat{A}_{R}\right]^{+} \tilde{X},
$$

where $\widehat{A}_{T}$ and $\widehat{A}_{R}$ stand for the estimates of $\mathbf{A}_{T}$ and $\mathbf{A}_{R}$ which have previously obtained, respectively. The LS fitting of equation (7) can be written as

$$
\min _{\mathbf{A}_{T}, \mathbf{A}_{R}, \mathbf{B}}\left\|\tilde{Y}-\left[\mathbf{A}_{R} \odot \mathbf{B}\right] \mathbf{A}_{T}^{T}\right\|_{F},
$$

where the noise signal is denoted by $\tilde{Y}$. The LS update of matrix $\mathbf{A}_{T}$ is written by

$$
\widehat{A}_{T}^{T}=\left[\widehat{A}_{R} \odot \widehat{B}\right]^{+} \widetilde{Y},
$$

where $\widehat{A}_{R}$ and $\widehat{B}$ on behalf of the estimates of $\mathbf{A}_{R}$ and $\mathbf{B}$ which have previously obtained, respectively. Similarly, the LS fitting of equation (8) can be written as

$$
\min _{\mathbf{A}_{T}, \mathbf{A}_{R}, \mathbf{B}}\left\|\widetilde{Z}-\left[\mathbf{B} \odot \mathbf{A}_{T}\right] \mathbf{A}_{R}^{T}\right\|_{F},
$$

where the noise signal is denoted by $\widetilde{Z}$. The LS update of matrix $\mathbf{A}_{R}$ is 


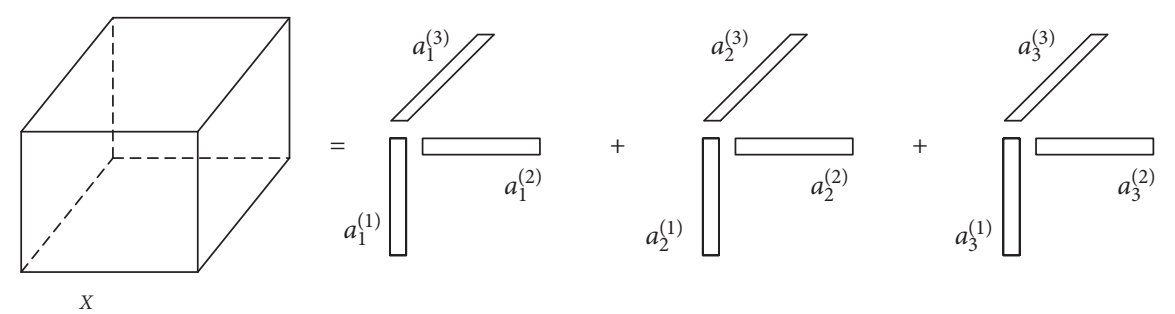

FIGURE 2: Schematic diagram of trilinear decomposition.

$$
\widehat{A}_{R}^{T}=\left[\widehat{B} \odot \widehat{A}_{T}\right]^{+} \widetilde{Z},
$$

where $\widehat{B}$ and $\widehat{A}_{T}$ on behalf of the estimates of $\mathbf{B}$ and $\mathbf{A}_{T}$ which have previously obtained, respectively.

According to equations (10), (12), and (14), $\mathbf{B}, \mathbf{A}_{T}$, and $\mathbf{A}_{R}$ are given by the LS. The LS update does not stop until convergence, and the constraint condition can be expressed as $\left\|\widetilde{X}-\left[\widehat{A}_{T} \odot \widehat{A}_{R}\right] \widehat{B}^{T}\right\|_{F}^{2} \leq 10^{-10}$.

For the received noise signals, according to the trilinear decomposition, we can get the estimated parameter matrices: $\quad \widehat{A}_{T}=\mathbf{A}_{T} \Lambda \mathbf{M}_{1}+\mathbf{N}_{1}, \quad \widehat{A}_{R}=\mathbf{A}_{R} \Lambda \mathbf{M}_{2}+\mathbf{N}_{2}$, and $\widehat{B}=\mathbf{B} \Lambda \mathbf{M}_{3}+\mathbf{N}_{3}$, where $\Lambda$ is a permutation matrix, $\mathbf{N}_{1}, \mathbf{N}_{2}$, and $\mathbf{N}_{3}$ stand for the matrices in correspondence to estimation errors, and $\mathbf{M}_{1}, \mathbf{M}_{2}$, and $\mathbf{M}_{3}$ denote the diagonal scaling matrices and are subject to $\mathbf{M}_{1} \mathbf{M}_{2} \mathbf{M}_{3}=\mathbf{I}_{K}$. The inherent scale ambiguity and permutation of trilinear decomposition and normalization can be used to eliminate scale ambiguity.

3.2. Angle Estimation. From the trilinear decomposition in the previous section, we can get estimates of the direction matrices $\widehat{A}_{T}$ and $\widehat{A}_{R}$ by the TALS method.

It can be seen from the signal model that the steer vector of $\widehat{A}_{R}$ is only related to the direction of arrival (DOA), and we can get the estimated value of the angle through $\widehat{A}_{R}$.

In the paper, the receive array can be preset as halfwavelengths spaced uniform linear arrays (ULA), so the receive steering vector of $\theta_{k}$ is

$$
\mathbf{a}_{r}\left(\theta_{k}\right)=\left[1, e^{\left(j \pi \sin \theta_{k}\right)}, \ldots, e^{\left(j \pi(N-1) \sin \theta_{k}\right)}\right]^{T} .
$$

Let $\psi_{r}$ denote the phase of $\mathbf{a}_{r}\left(\theta_{k}\right)$, which can be expressed as

$$
\psi_{r}=\operatorname{angle}\left(a_{r}\left(\theta_{k}\right)\right)=\left[0, \pi \sin \theta_{k}, \ldots, \pi(N-1) \sin \theta_{k}\right]^{T} .
$$

After the receiving steering vector is well calibrated, it is represented as $\hat{a}_{r}\left(\theta_{k}\right)$ and utilizes normalization to dispel scale ambiguity. So, $\widehat{\psi}_{r}$ can be obtained from equation (16). The estimate of $\sin \theta_{k}$ can be calculated by LS principle. We can construct the LS fitting as

$$
\mathbf{G} \mathbf{q}=\widehat{\psi}
$$

where the $\mathbf{q} \in \mathbb{C}^{2 \times 1}$ and $\widehat{\psi}$ stand for the estimated vector and the estimated phase of steering vector, $\mathbf{G}$ is defined as

$$
\mathbf{G}=\left[\begin{array}{cc}
1 & 0 \\
1 & \pi \\
\vdots & \vdots \\
1 & \pi(N-1)
\end{array}\right] \in \mathbb{C}^{N \times 2},
$$

$\widehat{q}_{r}$ is the LS solution of $\mathbf{q}_{r}$ by equation (17), which stands for the estimated vector and can be expressed as

$$
\widehat{q}_{r}=\left(\mathbf{G}^{T} \mathbf{G}\right)^{-1} \mathbf{G}^{T} \widehat{\psi}_{r} .
$$

The receive angle $\widehat{\theta}_{k}$ is derived from

$$
\widehat{\theta}_{k}=\sin ^{-1}\left(\widehat{q}_{r}(2)\right),
$$

where $\widehat{q}_{r}(2)$ represents the element in the second row of the vector $\widehat{q}_{r}$.

3.3. Range Estimation. For the tensor-based data model, the range and transmit angle of the uncorrelated target are both included in the transmit steering vector. So, the transmit steering vector can be expressed as

$$
\mathbf{a}_{t}\left(\theta_{k}, r_{k}\right)=\mathbf{a}_{t}\left(\theta_{k}\right) \circ \mathbf{a}_{t}\left(r_{k}\right) \text {, }
$$

where

$\mathbf{a}_{t}\left(\theta_{k}\right)=\left[1, e^{\left(-j 2 \pi d \sin \left(\theta_{k} / \lambda_{0}\right)\right)}, \ldots, e^{\left(-j 2 \pi d(M-1) \sin \left(\theta_{k} / \lambda_{0}\right)\right)}\right]^{T}$ and $\mathbf{a}_{t}\left(r_{k}\right)=\left[1, e^{\left(-j 2 \pi 2 \Delta f\left(r_{k} / c\right)\right)}, \ldots, e^{\left(-j 2 \pi 2 \Delta f(M-1) r_{k} / c\right)}\right]^{T}$ denote the transmitting angle steering vector and range steering vector, respectively.

The transmit array is preset as half-wavelengths spaced ULA, so the transmitting steer vector of $\left(\theta_{k}, r_{k}\right)$ can be reduced as

$$
\mathbf{a}_{t}\left(\theta_{k}, r_{k}\right)=\left[1, e^{j \pi\left(\sin \theta_{k}-4 \Delta f\left(r_{k} / c\right)\right)}, e^{j \pi(M-1)\left(\sin \theta_{k}-4 \Delta f\left(r_{k} / c\right)\right)}\right]^{T} .
$$

In the previous section, the direction of arrival (DOA) $\hat{\theta}_{k}$ has been obtained. Referring to the derivation process in the previous section and letting $\psi_{t}$ represent the phase of $\mathbf{a}_{t}\left(r_{k}, \theta_{k}\right)$, which can be expressed as

$$
\begin{aligned}
\psi_{t} & =\operatorname{angle}\left(a_{t}\left(\theta_{k}, r_{k}\right)\right) \\
& =\left[0, \pi\left(\sin \theta_{k}-4 \Delta f \frac{r_{k}}{c}\right), \ldots, \pi(M-1)\left(\sin \theta_{k}-4 \Delta f \frac{r_{k}}{c}\right)\right]^{T} .
\end{aligned}
$$

In the previous section, we have obtained the estimated receive vector $\widehat{q}_{r}$. Similarly, we can get $\widehat{q}_{t}$ by equation (17), 
which stands for the estimated transmit receive vector and can be expressed as

$$
\widehat{q}_{t}=\left(\mathbf{G}^{T} \mathbf{G}\right)^{-1} \mathbf{G}^{T} \widehat{\psi}_{t} .
$$

The range $\widehat{r}_{k}$ of target is derived from

$$
\widehat{r}_{k}=\frac{c\left(\sin \hat{\theta}_{k}-\widehat{q}_{t}(2)\right)}{4 \Delta f},
$$

where $\widehat{q}_{t}(2)$ represents the element in the second row of the vector $\widehat{q}_{t}$.

So far, we already get the angle and range of the uncorrelated target in the monostatic FDA-MIMO radar. We can recap the key processes of the proposed method as follows:

(i) Step 1: according to equation (6), the received data is transformed into a third-order tensor model.

(ii) Step 2: apply the trilinear decomposition principle; we can obtain the transmitting direction matrix $\widehat{A}_{T}$ and the receiving direction matrix $\widehat{A}_{R}$.

(iii) Step 3: take the phase $\widehat{q}_{r}$ and $\widehat{q}_{t}$ of $\widehat{A}_{R}$ and $\widehat{A}_{T}$ by equations (16) and (23), respectively.

(iv) Step 4: the phase $\widehat{\psi}_{r}$ of the receive direction matrix was plugged into equation (17) and obtain $\widehat{q}_{r}$ by LS fitting; then, the receive angle $\widehat{\theta}_{r}$ can be calculated by equation (20).

(v) Step 5: similarly, we can obtain $\widehat{q}_{t}$; then, the range $\widehat{r}_{k}$ of target can be calculated by substituting the angle into equation (25).

\section{CRB of FDA-MIMO Radar}

The input signal spectrum is defined as

$$
\mathbf{S}_{x}=\mathbf{A S}_{f} \mathbf{A}^{H}+\boldsymbol{\sigma}^{2} \mathbf{I}_{M N},
$$

where $\mathbf{S}_{f}$ is the unknown signal spectrum matrix. CRB formula can be expressed as $[17,32]$

$$
\mathrm{CRB}=\frac{\sigma^{2}}{2 L}\left\{\operatorname{Re}\left[\left(\left(\mathbf{S}_{f} \mathbf{A}^{H} \mathbf{S}_{x} \mathbf{A} \mathbf{S}_{f}\right) \otimes\left[\begin{array}{ll}
1 & 1 \\
1 & 1
\end{array}\right]\right) \circ\left(\mathbf{D}^{H} \mathbf{P}_{A}^{\perp} \mathbf{D}\right)^{T}\right]\right\}^{-1},
$$

where $\mathbf{D}=\left[\left(\left(\partial a\left(\theta_{1}, r_{1}\right)\right) / \partial \theta_{1}\right), \ldots,\left(\left(\partial a\left(\theta_{1}, r_{1}\right)\right) / \partial r_{1}\right), \ldots\right]$, and $\mathbf{P}_{A}^{\perp}=\mathbf{I}_{M N}-\mathbf{A}\left(\mathbf{A}^{H} \mathbf{A}\right)^{-1} \mathbf{A}^{H}$.

When $\left(\mathbf{A}^{H} \mathbf{A} \mathbf{S}_{f}\right) / \sigma^{2}$ is large enough, $\mathbf{S}_{f}$ and $\mathbf{A}^{H} \mathbf{A}$ are not close to singularity and the signals are unknown; the above formula can be approximated as an approximate CRB:

$$
\mathrm{CRB}_{A C R}=\frac{\sigma^{2}}{2 L}\left\{\operatorname{Re}\left[\left(\mathbf{S}_{f} \otimes\left[\begin{array}{ll}
1 & 1 \\
1 & 1
\end{array}\right]\right) \circ\left(\mathbf{D}^{\mathrm{H}} \mathbf{P}_{\mathrm{A}}^{\perp} \mathbf{D}\right)^{\mathrm{T}}\right]\right\}^{-1} .
$$

\section{Simulation Results}

In this section, the effectiveness and advantages of our proposed method can be proved by some numerical simulations. The ESPRIT method and Unitary-ESPRIT method can be utilized to contrast with our proposed method. We default the monostatic FDA-MIMO radar with $M=8$ transmitting antennas and $N=8$ receiving antennas in this paper.

Unless otherwise specified, it is supposed that there are $K=3$ uncorrelated targets which are objected to far-field. The three targets are placed at $\left(\theta_{1}, r_{1}\right)=\left(-15^{\circ}, 0.5 \mathrm{~km}\right)$, $\left(\theta_{2}, r_{2}\right)=\left(10^{\circ}, 6 \mathrm{~km}\right)$, and $\left(\theta_{3}, r_{3}\right)=\left(35^{\circ}, 80 \mathrm{~km}\right)$. The performance of our proposed method can be appraised by the root mean square error (RMSE), which can be expressed as

$$
\begin{aligned}
\operatorname{RMSE}_{\theta} & =\sqrt{\frac{1}{K} \frac{1}{T} \sum_{k=1}^{K} \sum_{t=1}^{T}\left(\widehat{\theta}_{k, t}, \theta_{k}\right)^{2},} \\
\operatorname{RMSE}_{r} & =\sqrt{\frac{1}{K} \frac{1}{T} \sum_{k=1}^{K} \sum_{t=1}^{T}\left(\widehat{r}_{k, t}, r_{k}\right)^{2}},
\end{aligned}
$$

where $\widehat{\theta}_{k, t}$ and $\widehat{r}_{k, t}$ are the estimation of DOA $\theta_{k}$ and range $r_{k}$ of the $k$ th target for the $t$ th Monte Carlo trials, respectively, $T$ denotes the total amount of Monte Carlo trials, and $T=500$ is preset in this simulation.

In addition, the probability of successful detection is another metric used to appraise the achievement of our method, which is defined by

$$
\mathrm{PSD}=\frac{V}{T} \times 100 \%,
$$

where $V$ represents the number of successful estimates, and the criterion for the success of the angle and range experiments is that the absolute value of all experimental numerical errors are less than the minimum $0.1^{\circ}$ and $0.1 \mathrm{~km}$, respectively.

We preset $\mathrm{SNR}=20 \mathrm{~dB}$ in the first simulation; Figure 3 shows the estimation results of our proposed method. Figure 3 shows the estimation of the range and angle of the uncorrelated targets are correct, and the landing points are highly concentrated. It directly proves the stability and accuracy of the proposed method.

And then, $L$ denotes the number of snapshots, and it is preset to $L=50$ in this simulation. We first investigate the liaison between RMSE and SNR of range estimation and angle estimation in the second simulation. We use two comparison methods, which are the ESPRIT method [31] and the Unitary ESPRIT method [32], respectively. After introducing $\mathrm{CRB}$, they are compared with the proposed method. Figures 4 and 5 correspond to the simulation. From the two figures, it can be demonstrated that the estimated performance of the proposed method is better than the ESPRIT method and the Unitary ESPRIT method. In addition, the RMSE of the proposed method is closer to CRB. 


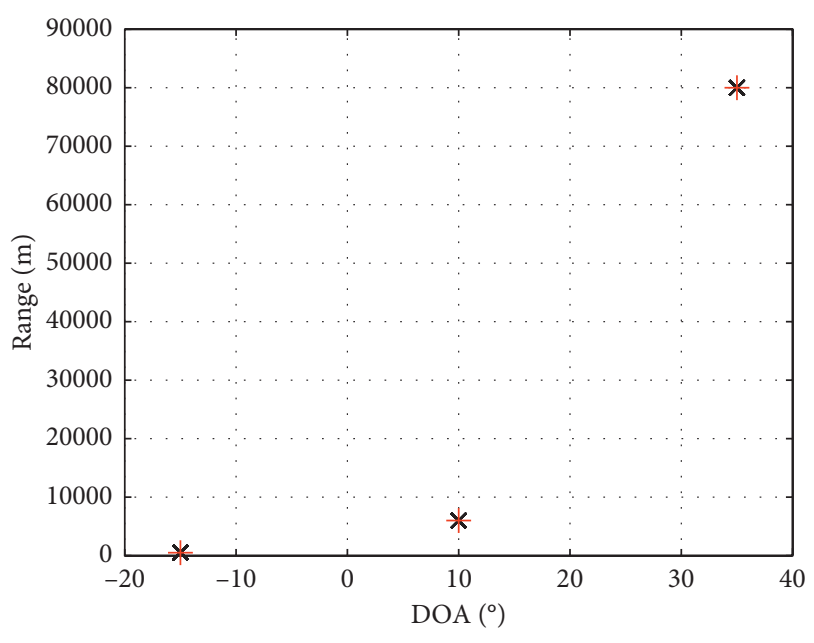

FIgURE 3: The range and angle estimation performance of the proposed method with $\mathrm{SNR}=20 \mathrm{~dB}$.

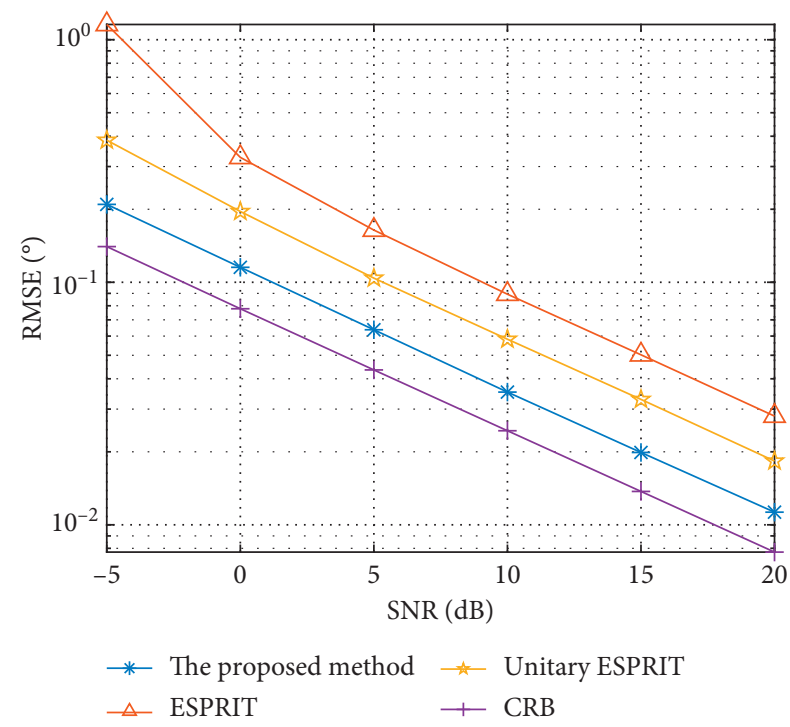

Figure 4: RMSE of angle estimation versus SNR.

From the following, we explore the relationship between RMSE and snapshots of range estimation and angle estimation in the third simulation, and the results are shown in Figures 6 and 7, respectively. The SNR = $10 \mathrm{~dB}$ is preset in this simulation. Similar to the first simulation, after introducing CRB, we use the contrast methods to compare with the proposed algorithm. As the number of snapshots increase, we can know from the figures that the RMSE of three methods and CRB all decrease. So, we conclude that the accuracy of the methods is improving, and the proposed method has the best performance.

In the fourth simulation, the relationship between probability and SNR of angle and range probability

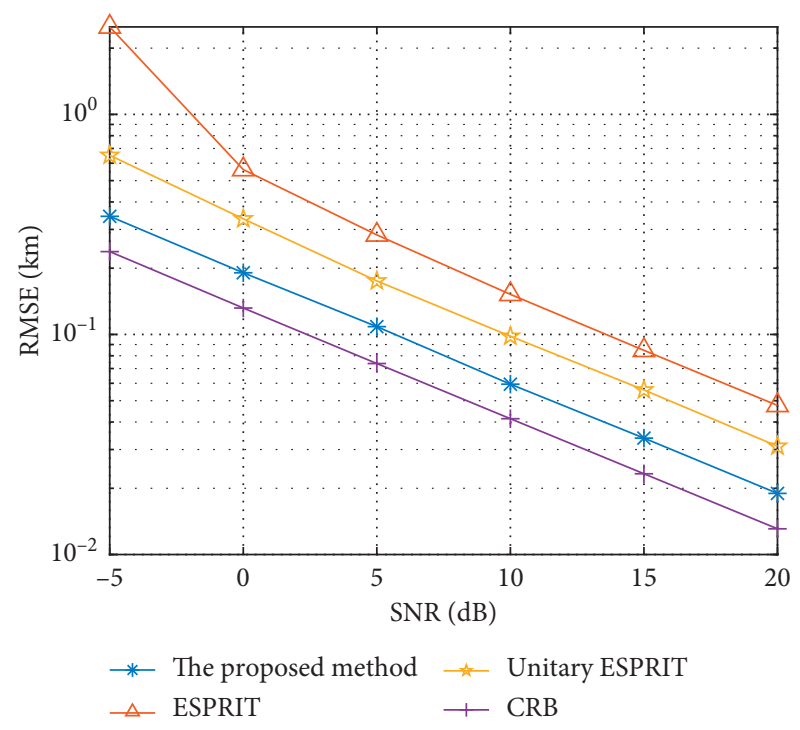

Figure 5: RMSE of range estimation versus SNR.

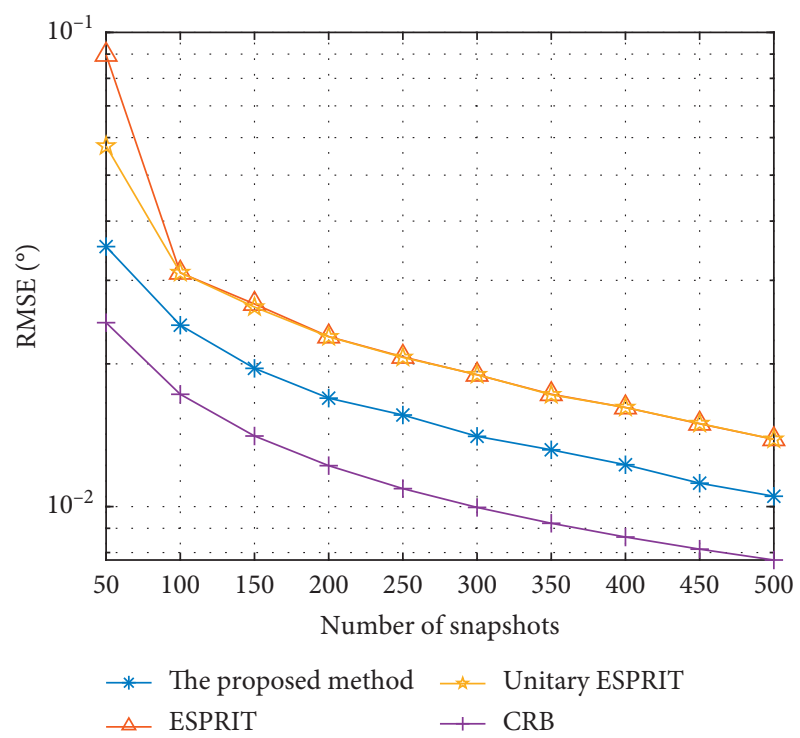

FIGURE 6: RMSE of angle estimation versus the total amount of snapshots.

successful detection is obtained. The number of snapshots is preset to $L=50$. Figures 8 and 9 correspond to the fourth simulation. From two figures, we can know the success rate of three methods can reach $100 \%$ with the increase of SNR. Moreover, with the increase of SNR, the probability of successful detection also increases. It can reach a detection success rate of $100 \%$, when the SNR reaches a sufficiently high level, this SNR is generally called the SNR threshold. Obviously, the threshold of the proposed method is the lowest among the three methods, which also illustrates the superiority of the proposed method. 


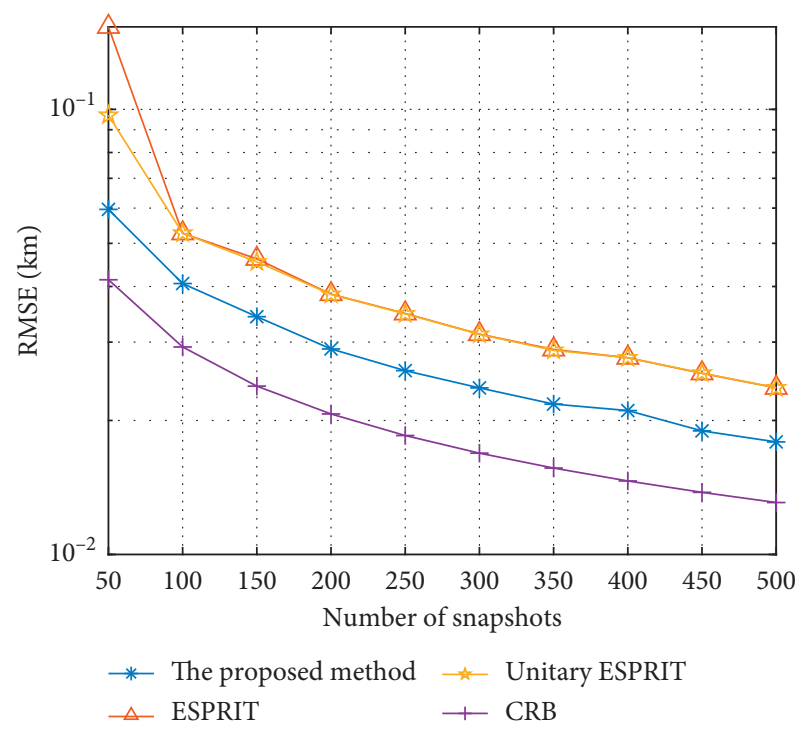

FIGURE 7: RMSE of range estimation versus the total amount of snapshots.

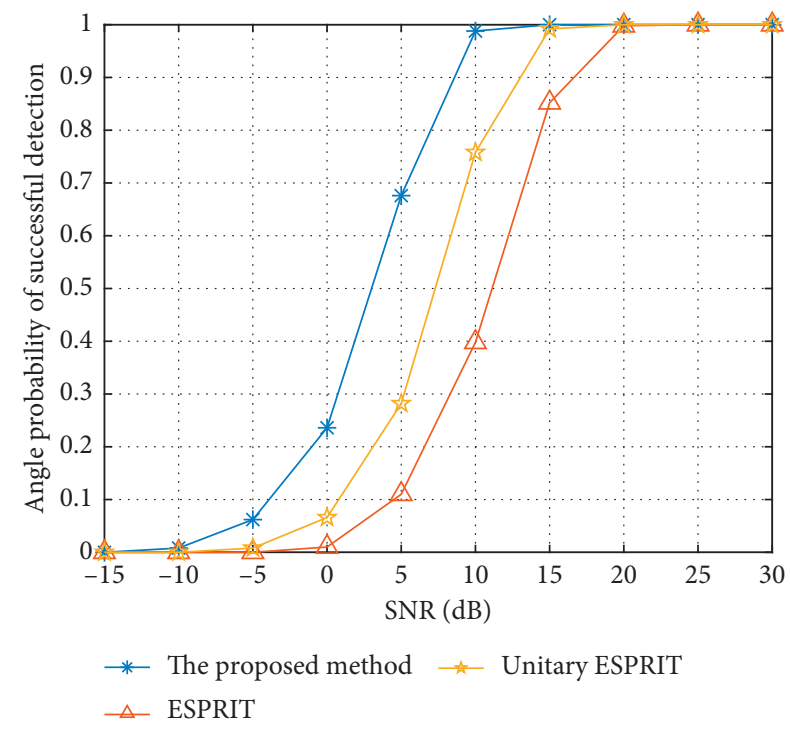

FIgURE 8: Probability of angle successful detection versus SNR.

\section{Conclusions}

In the paper, we proposed a tensor-based range and angle estimation method in monostatic FDA-MIMO radar. The proposed method uses the trilinear model to obtain the direction matrices through PARAFAC decomposition and extracts the phase from the direction matrix to estimate the distance and angle. This method uses the multidimensional information of the received data. Compared with the subspace methods such as ESPRIT and Unitary-ESPRIT methods, the proposed method has the best performance. The superiority of the proposed method can be verified by simulation.

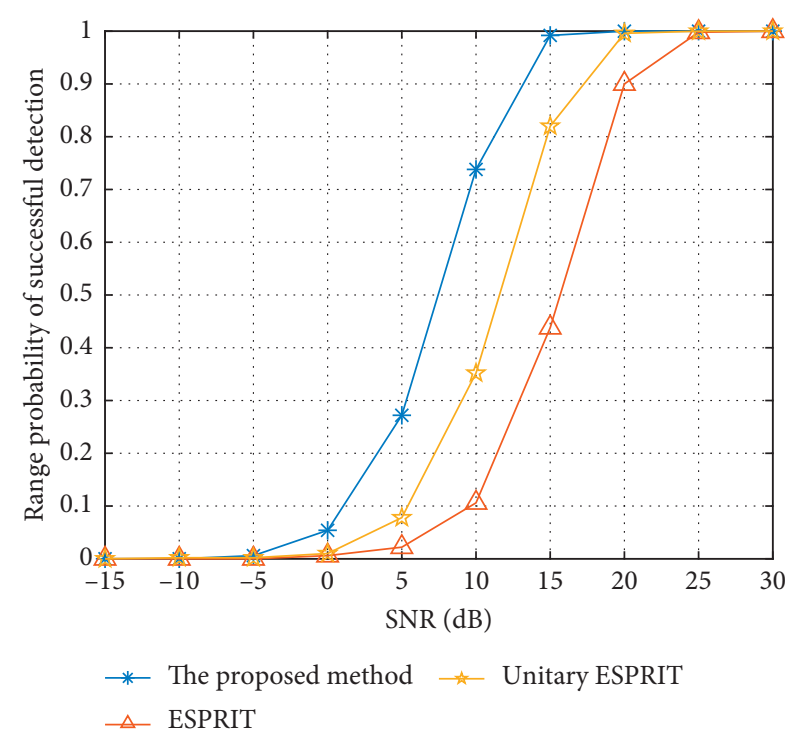

FIgURE 9: Probability of range successful detection versus SNR.

\section{Data Availability}

No data were used to support this study.

\section{Conflicts of Interest}

The authors declare that they have no conflicts of interest.

\section{Acknowledgments}

This work was supported by the Hainan Provincial Natural Science Foundation of China (no. 2018CXTD336) and National Natural Science Foundation of China (no. 61864002).

\section{References}

[1] E. Fishler, A. Haimovich, R. Blum et al., "MIMO radar: an idea whose time has come," in Proceedings of the IEEE Radar conference, vol. 7, pp. 71-78, Philadelphia, PA, USA, April 2004.

[2] H. Krim and M. Viberg, "Two decades of array signal processing research: the parametric approach," IEEE Signal Processing Magazine, vol. 13, no. 4, pp. 67-94, 1996.

[3] E. Fishler, A. Haimovich, R. Blum et al., "Performance of MIMO radar systems: advantages of angular diversity," in Proceeding of the Thirty-Eighth Asilomar Conference on Signals, Systems and Computer, vol. 1, pp. 305-309, Pacific Grove, CA, USA, November 2004.

[4] J. Li and P. Stoica, "MIMO Radar with colocated antennas," IEEE Signal Processing Magazine, vol. 24, no. 5, pp. 106-114, 2007.

[5] A. A. Gorji, R. Tharmarasa, T. Kirubarajan et al., "Optimal antenna allocation in MIMO radars with collocated antennas," IEEE Transactions on Aerospace and Electronic Systems, vol. 50, no. 1, pp. 542-558, 2014.

[6] A. Hassanien and S. A. Vorobyov, "Phased-MIMO radar: a tradeoff between phased-array and MIMO radars," IEEE 
Transactions Signal Processing, vol. 58, no. 6, pp. 3137-3151, 2009.

[7] E. Fishler, A. Haimovich, R. S. Blum, L. J. Cimini, D. Chizhik, and R. A. Valenzuela, "Spatial diversity in radars-models and detection performance," IEEE Transactions on Signal Processing, vol. 54, no. 3, pp. 823-838, 2006.

[8] A. Haimovich, R. Blum, L. Cimini et al., "MIMO radar with widely separated antennas," IEEE Signal Processing Magazine, vol. 25, no. 1, pp. 116-129, 2008.

[9] A. J. Fenn, D. H. Temme, W. P. Delaney et al., "The development of phased-array radar technology," Lincoln Laboratory Journal, vol. 12, no. 2, pp. 321-340, 2000.

[10] Y. Hua, T. K. Sarkar, D. D. Weiner et al., "An L-shaped array for estimating 2-D directions of wave arrival," IEEE Transactions on Antennas and Propagation, vol. 39, no. 2, pp. 143-146, 1991.

[11] X. Wang, L. Wan, M. Huang, C. Shen, and K. Zhang, "Polarization channel estimation for circular and non-circular signals in massive MIMO systems," IEEE Journal of Selected Topics in Signal Processing, vol. 13, no. 5, pp. 1001-1016, 2019.

[12] H. Wang, L. Wan, M. Dong, K. Ota, and X. Wang, "Assistant vehicle localization based on three collaborative base stations via SBL-based robust DOA estimation," IEEE Internet of Things Journal, vol. 6, no. 3, pp. 5766-5777, 2019.

[13] X. Wang, D. Meng, M. Huang, and L. Wan, "Reweighted regularized sparse recovery for DOA estimation with unknown mutual coupling," IEEE Communications Letters, vol. 23, no. 2, pp. 290-293, 2019.

[14] D. Meng, X. Wang, M. Huang, L. Wan, and B. Zhang, "Robust weighted subspace fitting for DOA estimation via block sparse recovery," IEEE Communications Letters, vol. 24, no. 3, pp. 563-567, 2020.

[15] P. Antonik, M. C. Wicks, H. D. Griffiths et al., "Frequency diverse array radars," in Proceedings of the 2006 IEEE Radar Conference, pp. 215-217, Verona, NY, USA, April 2006.

[16] P. F. Sammartino, C. J. Baker, H. D. Griffiths et al., "Frequency diverse MIMO techniques for radar," IEEE Transactions on Aerospace and Electronic Systems, vol. 49, no. 1, pp. 201-222, 2013.

[17] W.-Q. Wang and H. C. So, “Transmit subaperturing for range and angle estimation in frequency diverse array radar," IEEE Transactions on Signal Processing, vol. 62, no. 8, pp. 20002011, 2014.

[18] W.-Q. Wang, "Subarray-based frequency diverse array radar for target range-angle estimation," IEEE Transactions on Aerospace and Electronic Systems, vol. 50, no. 4, pp. 30573067, 2014.

[19] S. Qin, Y. D. Zhang, M. G. Amin, and F. Gini, "Frequency diverse coprime arrays with coprime frequency offsets for multitarget localization," IEEE Journal of Selected Topics in Signal Processing, vol. 11, no. 2, pp. 321-335, 2017.

[20] W.-Q. Wang and C. Zhu, "Nested array receiver with timedelayers for joint target range and angle estimation," IET Radar, Sonar \& Navigation, vol. 10, no. 8, pp. 1384-1393, 2016.

[21] S. Qin and Y. M. D. Zhang, "Frequency diverse array radar for target range-angle estimation," International Journal of Computations and Mathematics in Electrical, vol. 35, no. 3, pp. 1257-1270, 2016.

[22] P. F. Sammartino, C. J. Baker, H. D. Griffiths et al., "Rangeangle dependent waveform," in Proceedindgs of the IEEE Radar Conference, pp. 511-515, Washington, DC, USA, May 2010.
[23] Y.-Q. Yang, H. Wang, H.-Q. Wang, S.-Q. Gu, D.-L. Xu, and S.-L. Quan, "Optimization of sparse frequency diverse array with time-invariant spatial-focusing beampattern," IEEE Antennas and Wireless Propagation Letters, vol. 17, no. 2, pp. 351-354, 2018.

[24] A. M. Yao, W. Wu, D. G. Fang et al., "Frequency diverse array radar with time-dependent frequency offset," IEEE Antennas and Wireless Propagation Letters, vol. 13, pp. 758-761, 2014.

[25] D. Nion and N. D. Sidiropoulos, "Adaptive algorithms to track the PARAFAC decomposition of a third-order tensor," IEEE Transactions on Signal Processing, vol. 57, no. 6, pp. 2299-2310, 2009.

[26] D. Nion and N. D. Sidiropoulos, "Tensor algebra and multidimensional harmonic retrieval in signal processing for MIMO radar," IEEE Transactions on Signal Processing, vol. 58, no. 11, pp. 5693-5705, 2010.

[27] B. Xu, Y. Zhao, Z. Cheng, H. Li et al., "A novel unitary PARAFAC method for DOD and DOA estimation in bistatic MIMO radar," Signal Processing, vol. 138, no. 11, pp. 273-279, 2017.

[28] D. Nion and N. D. Sidiropoulos, "A PARAFAC-based technique for detection and localization of multiple targets in a MIMO radar system," in Proceedings of the IEEE international conference on Speech and Signal \& Processing, pp. 2077-2080, Taipei, Taiwan, April 2009.

[29] F. Wen, X. Xiong, and Z. Zhang, "Angle and mutual coupling estimation in bistatic MIMO radar based on PARAFAC decomposition," Digital Signal Processing, vol. 65, pp. 1-10, 2017.

[30] J. F. Li and X. F. Zhang, "A method for joint angle and array gain-phase error estimation in bistatic multiple-input multiple-output non-linear arrays," IET Signal Processing, vol. 8, no. 2, pp. 131-137, 2014.

[31] B. Li, W. Bai, G. Zheng et al., "Successive ESPRIT algorithm for joint DOA-range-polarization estimation with polarization sensitive FDA-MIMO radar," IEEE Access, vol. 6, pp. 36376-36382, 2018.

[32] F. Liu, X. Wang, M. Huang et al., "A novel unitary ESPRIT algorithm for monostatic FDA-MIMO radar," Sensors, vol. 20, no. 3, 827 pages, 2020.

[33] X. Zhang, Z. Xu, and D. Xu, "Trilinear decomposition-based transmit angle and receive angle estimation for multiple-input multiple-output radar," IET Radar, Sonar \& Navigation, vol. 5, no. 6, pp. 626-631, 2011. 